\title{
STATE VARIABLES MONITORING USING A CLASS OF NONLINEAR OBSERVER BASED ESTIMATOR, APPLIED TO CONTINUOUS BIO-SYSTEM
}

\author{
Ricardo Aguilar-López, Ricardo Acevedo-Gómez, María Isabel Neria González* and Alma Rosa \\ Domínguez-Bocanegra
}

\author{
Departamento de Biotecnología y Bioingeniería \\ CINVESTAV-IPN \\ Av. Instituto Politécnico Nacional No. 2508, San Pedro Zacatenco, México, D.F. 07360, MEXICO \\ E-mail.- raguilar@cinvestav.mx \\ Phone.- + 525557473800 , ext. 4307 \\ *División de Ingeniería Química y Bioquímica \\ Tecnológico de Estudios Superiores de Ecatepec \\ Av. Tecnológico s/n Esq. Av. Carlos Hank González Col. Valle de Anáhuac C.P. 55210, Ecatepec Estado de México
}

\begin{abstract}
In this work, the state estimation of key variables such as biomass and products of a sulfate reducing bacterium is predicted by using only sulfate (substrate) concentration measurements under the assumption of an unknown kinetic term. The process was developed by continuous culture, where the mathematical kinetic model for the biomass, sulfate and sulfide concentrations is presented and tuned using experimental data. The design of the nonlinear state estimator takes into account an adaptive gain. The results of the proposed estimation methodology were generated via numerical simulation; they showed a satisfactory performance.
\end{abstract}

KEYWORDS: robust estimation, bioreactor model, anaerobic biosystem

\section{RESUMEN}

En este trabajo se predice la estimación de estados como la biomasa y la concentración de productos de una bacteria sulfato reductora empleando únicamente mediciones de la concentración de sulfato (sustrato) bajo la hipótesis de una cinética de consumo de sulfato desconocida. El proceso fue considerado como un cultivo continuo, donde el modelo cinético correspondiente para las concentraciones de biomasa, sulfato y sulfuro fue corroborado con datos experimentales. El diseño de la metodología de estimación propuesta considera una ganancia adaptable. Los resultados de estimación fueron generados por medio de simulaciones numéricas y muestran un comportamiento satisfactorio.

Palabras Clave: Estimación robusta, modelo de un birreactor, bio-sistema anaerobio

\section{INTRODUCTION}

The lack of cheap and reliable instrumentation for the online measurement of the relevant variables in many processes definitely constitutes a serious obstacle for the development of biological systems. One way to overcome this problem is to use software sensors. A software sensor can be described as the relationship between sensor hardware and estimator software. Biological processes have become widely used in the industry for the last decades, with different purposes: either to produce some chemical compounds synthesized by a microorganism to cultivate a biomass for its utilization or extraction of its metabolites or to degrade a pollutant. Therefore, bioreactors require advanced monitoring procedures to ensure the performances and efficiency bioprocesses operation [1, 2]. 
The estimation of the key variables such as specific substrate consumption rates, specific microbial growth rates, products and biomass concentrations are very valuable tools to analyze the performance of biotechnological systems. In particular, sulfate-reducing bacteria (SRB) are anaerobic microorganisms of great ecological importance in the global carbon and sulfur cycles, they oxidize the organic compounds coupled to the reduction of sulfate; therefore, they are used on anaerobic processes for wastewater treatment [3-5].

The sulfate-reducing bioreactors have been constructed from some biotechnological processes, as mentioned above, in which the monitoring of increases in the cell mass and products is difficult to measure given the anaerobic process conditions. Different methods for detection and enumeration of SRB in natural and industrial environments have been developed; they have been grouped in (i) direct detection methods and (ii) culture methods [6]. The direct detection involved the use of antibodies raised against SRB [7] and the use of molecular biology tools such as the 16S rRNA analysis; both techniques may be used in situ but required of a bigger knowledge and, in some cases, their use was not possible because of the nature of the considered sample. Culture methods for enumeration of SRB require of strict anaerobic conditions and special culture medium, experience of handling of these bacterial, the incubation times are sometimes very large, etc.[8].

Following these ideas, the estimation theory deserves an interesting research field because the estimation methodologies developed are widely employed in on-line monitoring, fault detection, control process and so on. Some of the most important estimation methodologies are related with the observers design where nonlinear Luenberger-type filters, Kalman filters, sliding-mode observers, and so on [9-13] have been presented in the open literature. On the other hand, some techniques such as neuralnetworks have been successfully used too $[13,14]$. Previous published work on observer design also include finite time observers, algebraic estimators, observers with modeling uncertainties and so on [1518]; however, several of these methodologies are developed on a complex mathematical frame and, consequently, the possibility of on-line implementation is hard. In this paper, it is considered the application of a class of state observer structure based on uncertainty estimator in order to estimate the key variables as biomass concentration and sulfide production in a continuous culture of Desulfovibro alaskensis, where an adaptive gain based on a fractional power of the absolute value of the estimation error is considered in the observer structure. This adaptive gain coupled with a proportional term of the estimation error provides asymptotic and exponential convergence; this proposed structure looks very simple and the possible real time implementation would be feasible.

\section{EXPERIMENTAL}

Desulfovibrio alaskensis 6SR strain was isolated of an oil pipeline [19]. Previously, the strain was cultured in Ravot medium [20] for 15 days at $32{ }^{\circ} \mathrm{C}$ under an atmosphere of N2-CO2 (80:20, v/v). Congenital water medium (CW). A sample of congenital water was obtained from an oil pipeline located in the Mexican southeast region. Chemical determination of water: chlorides 64000 ppm, sulfur 178 ppm, sulfate 350 to $400 \mathrm{ppm}, \mathrm{pH} 8.84$. A $1000 \mathrm{~mL}$ aliquot of congenital water was saturated whit N2 for 1 hour and was enriched with sodium lactate $6 \mathrm{~mL}$, yeast extract $0.5 \mathrm{~g}$, and reducing solution $5 \mathrm{~mL}$ (acid ascorbic $1 \mathrm{~g} / \mathrm{L}$, and sodium thioglicolate, $1 \mathrm{~g} / \mathrm{L}$ ). The $\mathrm{pH}$ was adjusted to 7 with $\mathrm{KOH} 1 \mathrm{~N}$. The $\mathrm{CW}$ medium was distributed in $60 \mathrm{~mL}$ serum bottles using the Hungate technique [21] and they were autoclaved at $120^{\circ} \mathrm{C}$ for $15 \mathrm{~min}$. The cultures initiate from $D$. Alaskensis in medium Ravot were used to inoculate $45 \mathrm{~mL}$ of $\mathrm{CW}$ medium. The culture was incubated for 20 days to $37^{\circ} \mathrm{C}$. This was used to inoculate three bottles with $\mathrm{CW}$ medium to different time: zero, 24 and 36 hours, respectively, and were incubated under same conditions. The bacterial growth was followed through Optical Density (OD). Samples from the cultures were taken anaerobically each hour. Sulphate in the medium was measured by the turbidimetric method based on the precipitation of barium [22]. Also, the production of sulfide was measured by a turbimetric method [23]. The OD reading for cell growth was transformed to dry weight $(\mathrm{mg} / \mathrm{mL})$ through a standard curve of growth. 


\section{MATHEMATICAL MODEL OF THE BIOREACTOR}

Anaerobic bioreactors are large fermentation tanks provided with mechanical mixing, heating, gas collection, sludge addition and withdrawal ports, and supernatant outlets that can be considered as continuous stirred tanks for analysis purposes. However, for estimation purposes, a reduced order model which can describe the dynamic behavior of the main state variables is adequate. The kinetic parameters were determinated via standard methodology [24] in a batch culture.

If the specific growth rate follows a Monod model, i. e.:

$\mu(S)=\mu_{\max } \frac{S}{k_{S}+S}$.The corresponding kinetic parameters can be fitted by plotting $\mu^{-1}$ versus $S^{-1}$.

Therefore,

$\mu(S)=\frac{0.035 S}{0.9+S}$

with $Y_{S / X}=0.25$ and $Y_{P / X}=0.263$.

Considering the above kinetic model, it is proposed the following mathematical model for a class of continuous stirred bioreactor based on classical mass balances for biomass, sulfate (substrate) and sulfide (product) concentrations:

Biomass $(\mathrm{X})$--

$\frac{d X}{d t}=-D X+\mu(S) X$

Sulfate (S).-

$\frac{d S}{d t}=D\left(S_{i n}-S\right)-\mu(s) \frac{X}{Y_{S / X}}$

Sulfide $(P)$ -

$$
\frac{d P}{d t}=-D P+\mu(S) \frac{X}{Y_{P / X}}
$$

Here $D$ is the dilution rate, $\mu$ is the specific growth rate, $Y_{S I X}$ is the sulfate coefficient yield and $Y_{P I X}$ is the sulfide coefficient yield. In accordance with the specific experimental setup, the following initial conditions are considered for the batch culture and model validation purposes: $X_{0}=0.12 \mathrm{~g} / \mathrm{L}, \mathrm{S}_{0}=5 \mathrm{~g} / \mathrm{L}, \mathrm{P}_{0}=0.16$ $\mathrm{g} / \mathrm{L}$. Figure. 1 shows the performance of the kinetic model considering a comparison with the experimental data which looks satisfactory. 


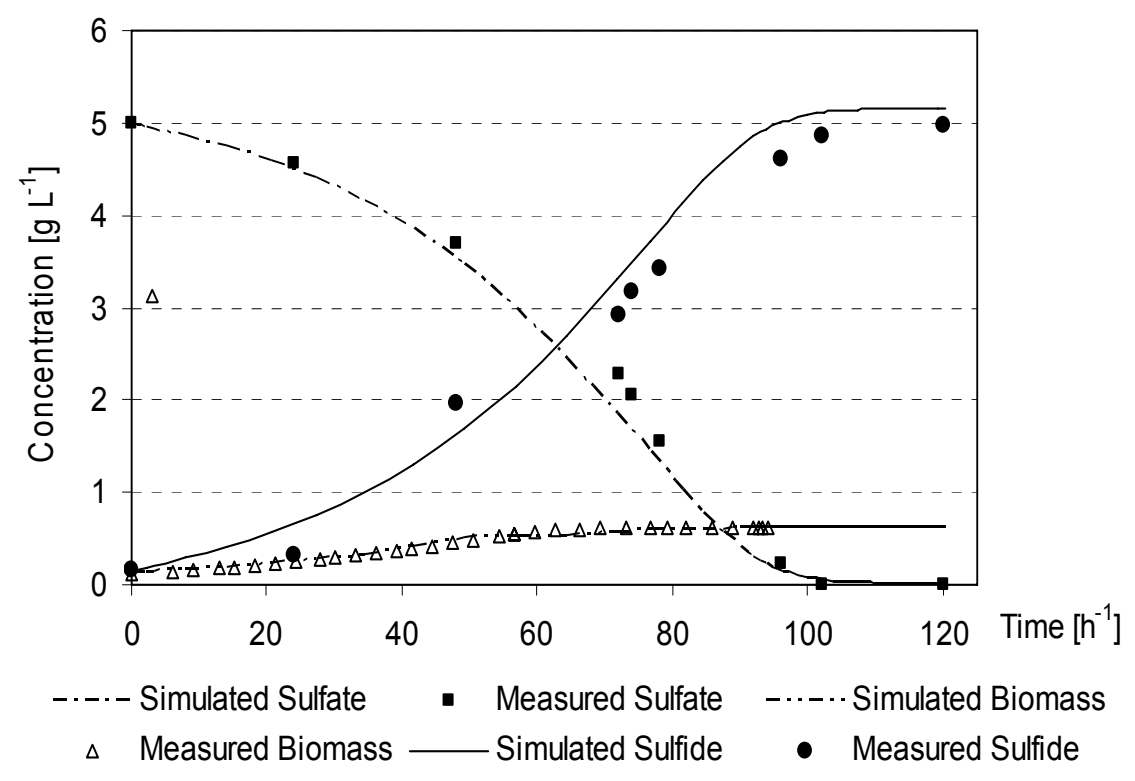

Figure 1. Kinetic model validation with experimental data.

\section{METHODOLOGY FOR THE OBSERVER DESIGN}

Consider a canonical form of the bioreactor model:

$$
\begin{aligned}
& \dot{x}=f(x)+g(x) u \\
& y=h(x)=C x
\end{aligned}
$$

Here, $x \in \mathfrak{R}^{n}$ is the vector of states; $u \in \mathfrak{R}^{q}$ is the vector control input; $f(\circ): \mathfrak{R}^{n} \rightarrow \mathfrak{R}^{n}$ is a nonlinear, partially known vector field; $g(\circ): \mathfrak{R}^{n} \rightarrow \mathfrak{R}^{n}$ is a linear vector of arguments and $y \in \mathfrak{R}^{m}$ is the system measured output. Now, consider the following assumptions:

A1. The system given by Equations (1a) and (1b) is locally uniformly observable, hence, for all, $x \in \mathfrak{R}^{n}$ and $\mathrm{u} \in \mathfrak{R}^{q}$ satisfies the observability rank condition:

$\operatorname{rank}\left\{\frac{\partial}{\partial x} \vartheta\right\}=n$

Here $\vartheta$ is the observability vector function defined as

$\vartheta=\left(d L_{f}^{0} h_{1}, \ldots, d L_{f}^{0} h_{m}, \ldots, d L_{f}^{1} h_{1}, \ldots, d L_{f}^{1} h_{m}, \ldots, d L_{f}^{n-1} h_{1}, \ldots, d L_{f}^{n-1} h_{m}\right)^{T}$, being $L_{f}^{r} h_{s}(\mathrm{~s}=1,2, \ldots, \mathrm{m})$ the r-order Lie derivatives. 
Now, consider $g(x)=\bar{g}(x)+\Delta g(x)$, and functions $f(x)$ and $\Delta g(x)$ are model uncertainties related to the non-linear system, $\bar{g}(x)$ is a nominal value of the control input coefficient. In the most general case, functions $f(x)$ and $\Delta g(x)$ are assumed to be unknown. Defining:

$$
\zeta(x, u)=f(x)+\Delta g(x) u
$$

By introducing (6) into (5), a new representation of the system is obtained (Equation 7).

$$
\begin{aligned}
& \dot{x}=\zeta(x, u)+\bar{g}(x) u \\
& \dot{\zeta}=\Phi(x, u) \\
& y=h(x)=C x
\end{aligned}
$$

In order to simplify notation, this set of equations can be written in vector notation (Equation 8).

$$
\begin{aligned}
& \dot{\Gamma}=\Theta \\
& y=C \Gamma
\end{aligned}
$$

Here $\Gamma=\left[\begin{array}{c}x \\ \zeta\end{array}\right] ; \Theta=\left[\begin{array}{c}\zeta(x, u)+\bar{g}(x) u \\ \Phi(x, u)\end{array}\right]$. The procedure described below provides a method to estimate the

uncertainty term, $\zeta(x, u)$. Estimators or observers for states and uncertainties can play a key role during the early detection of hazardous and unsafe operating conditions. Following this spirit, several researches have been focused in the proposition of estimation methodologies for states and uncertainties for monitoring and control purposes [25-28].

Now, the following state observer is proposed:

$$
\begin{aligned}
& \dot{\hat{x}}=\zeta(\hat{x}, u)+\bar{g}(\hat{x}) u+k_{1}(y-\hat{y}) \\
& \dot{\hat{\zeta}}=k_{2}(y-\hat{y}) \\
& \dot{K}=-\lambda \mid(y-\hat{y})^{1 / m}
\end{aligned}
$$

By defining $\hat{\Gamma}=\left[\begin{array}{l}\hat{x} \\ \zeta\end{array}\right], \hat{\Theta}=\left[\begin{array}{c}\hat{\zeta}(\hat{x}, u)+\bar{g}(\hat{x}) u \\ 0\end{array}\right]$ and $K=\left[\begin{array}{ll}k_{1} & k_{2}\end{array}\right]$, Equation (9) can be rewritten as 


$$
\begin{aligned}
& \dot{\hat{\Gamma}}=\hat{\Theta}+K(y-\hat{y}) \\
& \dot{K}=-\lambda \mid(y-\hat{y})^{1 / m}
\end{aligned}
$$

Here the dynamic equation for $K$ is an adaptation algorithm that updates the time-varing observer gain and $\beta$ is a parameter design.

In order to prove the convergence of the proposed observer, lets consider the dynamic equation of the estimation errors, $\varepsilon=\Gamma-\hat{\Gamma}$, as follows:

$$
\begin{aligned}
& \dot{\varepsilon}=\mathfrak{J}-\mathfrak{J}+K \varepsilon \\
& \dot{K}=-\lambda|\varepsilon|^{1 / m}
\end{aligned}
$$

Because the error is a finite quantity, there should be a constant $L$ that

A2. $|\Theta-\hat{\Theta}| \leq L|\Gamma-\hat{\Gamma}|$

Taking norms to both sides of equation (11) and applying A2, it is obtained

$$
|\bullet \dot{\varepsilon}| \leq L|\varepsilon|+K|\varepsilon|
$$

Now, to solve the system given by Equation (11), consider function $|\varepsilon|$ as a positive continuous function on the integration interval $[a, b]$; if $\Psi$ is the maximum of the function on the domain $[a, b]$, then $|\varepsilon|$ is bounded, i.e. $|\varepsilon| \leq \Psi \quad \forall t \in[a, b]$, hence

$$
|\varepsilon|^{1 / m} \leq \Psi^{1 / m} \quad m>0 \Rightarrow \int_{a}^{b}|\varepsilon|^{1 / m} \leq \Psi^{1 / m}(b-a)
$$

Here, $m$ is restricted to be an odd number i.e. $m=2 p+1, p \in Z^{+}$. Therefore, for $p$ large enough, the following limit is obtained:

$\lim \sup \int_{a}^{b}|\varepsilon|^{1 /(2 p+1)} \leq \lim \sup \Psi^{1 /(2 p+1)}(b-a) \leq(b-a)$

Applying the equality $|\varepsilon|=\operatorname{sign}(\varepsilon) \varepsilon$ to equation (12), another quota can be found

$\operatorname{sign}(\dot{\varepsilon}) \dot{\varepsilon} \leq(L-\lambda(b-a)) \operatorname{sign}(\varepsilon) \varepsilon$

By solving Equation (15), it is possible to note that the error is bounded by 
$\varepsilon \leq \varepsilon_{0} \exp \left(\operatorname{sign}(\dot{\varepsilon})^{-1} \operatorname{sign}(\varepsilon)(L-\lambda(b-a)) t\right)$

Therefore, the tracking error will be asymptotically and exponentially stable if

$\lambda>L(b-a)^{-1}$

\section{NUMERICAL EXPERIMENTS AND DISCUSSION}

A mathematical model of the cellular growth of a sulphate-reducing bacterium (Desulfovibrio alaskensis) is presented; this model is employed as a real system for the proposed observer design where the numerical simulation of the ordinary differential equations is done via ode23s Math labß library reproducing adequately the corresponding experimental data. The proposed estimation procedure considers the sulfate concentration (substrate) as measured output in order to infer the biomass, sulfide concentrations and the sulfate consumption rate (uncertain term), which are observable. The observer takes into account the following initial conditions: ( $X_{0}=0.1 \mathrm{~g} / \mathrm{L}$, So $=5 \mathrm{~g} / \mathrm{L}, P_{o}=0.16 \mathrm{~g} / \mathrm{L}, \zeta o=1.0 \mathrm{~g} / \mathrm{L} \mathrm{h}$ ) and for illustration purposes, a set of nominal operation conditions is considered $\left(D=0.251 /\right.$ hour and $S_{\text {in }}$ $=5 \mathrm{~g} / \mathrm{L}$ ) and the observer gain of $\lambda=0.0051 / \mathrm{hr}$. Figure 2 is related to the uncertainty (substrate consumption rate) estimation, as can be seen, a satisfactory performance is reached; from the start of the estimation procedure a small overshoot is presented, however, at 35 hours the uncertainty estimator faster converges to the real value. Figure 3 shows the states (sulfate, biomass and sulfite concentrations) estimation for the sulfate concentration estimation note an improved convergence; this is due to the fact thtat the sulfate concentration is the corresponding measured output. For the other concentrations, it is observed a fast convergence to the real trajectory, with a small offset which can be diminished via high values of the observer's gain.

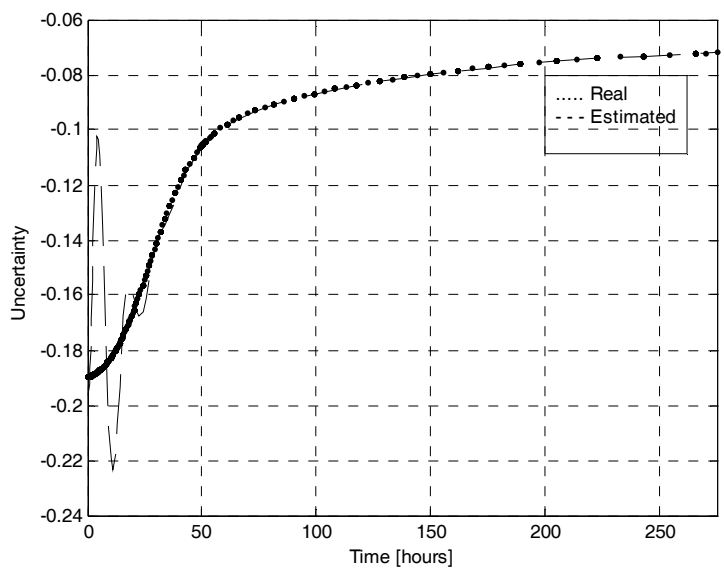

Figure 2. Uncertainty estimation performance

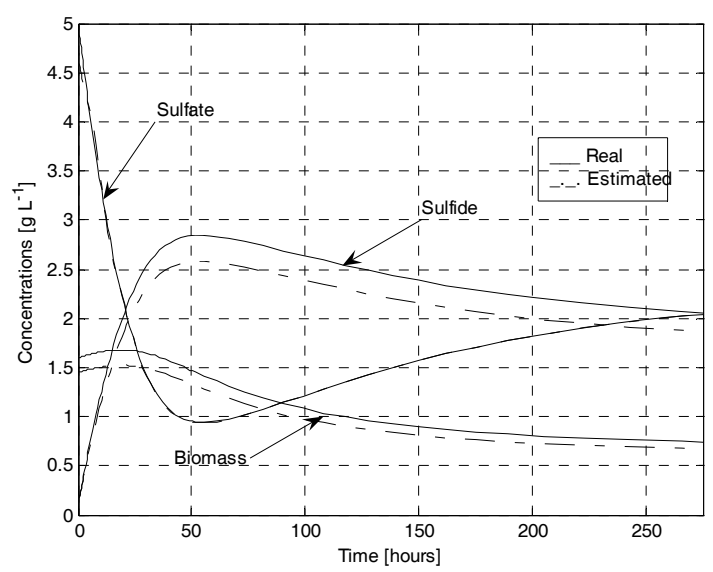

Figure 3. State estimation performance

\section{CONCLUSIONS}

In this work, the cellular growth, sulphate consumption and sulfide production are modeled for Desulfovibrio alaskensis using a classical mass balance and the Monod kinetic models. This model is compared with the experimental data successfully; therefore, this is employed as the real process for observer implementation purposes. An exponential-type state observer, coupled with an uncertainty estimator, is implemented for a continuous stirred bioreactor via numerical simulations to infer biomass and sulfide concentration and the sulfate consumption rate (uncertain term) from sulfate concentration 
measurements. A theoretical frame is provided in order to show the exponential convergence properties of the proposed methodology. Numerical experiments allow observing a satisfactory estimation performance, avoiding the experimental methods for detection and enumeration of SRB and sulfide production. The mathematical analysis to show the convergence characteristics of the proposed methodology is done. The proposed nonlinear observer based estimator would be employed for monitoring, fault detection and control purposes.

\section{ACKNOWLEDGEMENTS}

R. Acevedo is very grateful to CONACyT for the financial support via a postgraduate scholarship.

\section{NOMENCLATURE}

D.- Dilution rate [1/hour]

L.- Lipschitz constant [1/hour]

u.- Control input [1/hour]

t.- Time [hours]

K.- Adaptive observer vector gain [1/hour]

$X$.- Biomass concentration $[\mathrm{g} / \mathrm{L}]$

S.- Sulfate concentration $[\mathrm{g} / \mathrm{L}]$

$S_{\text {in.- }}$ Inlet Sulfate concentration [g/L]

Sign.- Discontinuous function with values [-1 1 1]

m.- Observer parameter

$P$.- Sulfide concentration $[\mathrm{g} / \mathrm{L}]$

$x$.- State variables vector

y.- Measured output

$Y_{S / X}$ - Sulfate yield coefficient

$Y_{P / X}$ - Sulfide yield coefficient

Greek Letters :

E.- Estimation error

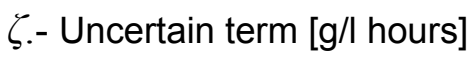

$\lambda$ - Observer gain [1/hour]

$\mu$.- Specific growth rate [1/hour]

$\mu_{\max }$ - Maximum specific growth rate [1/hour]

Г.- Estimation matrix 


\section{REFERENCES}

[1] J. González, G. Fernández, R. Aguilar, M. A. Barrón and J. Alvarez-Ramírez. Sliding mode observer-based robust control for a class of bioreactors. The Chemical Engineering Journal. 2001, 83, 25-32.

[2] Daniel R. Omstead (Editor). Computer control of fermentation process. CRC Press Inc. Florida, 1990.

[3] Devereux, R., He, S. H., Doyle, C. L., Orkland, S., Stahl, D. A., LeGall, J. \& Whitman, W. B. (1990). Diversity and origin of Desulfovibrio species: phylogenetic definition of a family. $J$ Bacteriol 172, 3609-3619.

[4] Feio, M. J., Beech, I. B., Carepo, M. \& 8 other authors (1998). Isolation and characterization of a novel sulphate-reducing bacterium of the Desulfovibrio genus. Anaerobe 4, 117-130.

[5] Feio MJ, Zinkevich V, Beech IB, Llobet-Brossa E, Eaton P, Schmitt J and Guezennec J. Desulfovibrio alaskensis sp. nov., a sulphate-reducing bacterium from a soured oil reservoir. Int. J. Syst. Evol Microbiol. 54 (2004) 1747-52.

[6] Vester F and Ingvorsen K. Improved most-probable-number method to detect sulfate reducing bacteria with natural media and a radiotracer. Appl. Environ. Microbiol. 64 (1998) 1700-1707.

[7] Lane, D. J. (1991). 16S/23S rRNA sequencing. In Nucleic Acid Techniques in Bacterial Systematics, pp. 115-175. Edited by E. Stackebrandt \& M. Goodfellow. Chichester: Wiley.

[8] American Public Health. Associated. Estimation of bacterial density. In: standard methods for the examination of water and waste-water, $17^{\text {th }}$ ed. America Public Health, Association Washington D.C. (1989) 977-980.

[9] Ricardo Aguilar, Jesús González, José Alvarez and Miguel Angel Barrón. Temperature regulation of a class of continuous chemical reactor based on a nonlinear Luenberger-like observer. Journal of Chemical Technology and Biotechnology. 1997. Vol. 70, No. 3, 209-216.

[10] Thau FE. Observing state of nonlinear dynamic systems. Int. J. Control. 17 (1973) 471-479.

[11] Ruijun Z, Tianyou C and Cheng S. Robust nonlinear observer design using dynamic recurrent neural networks. Proc. of the IEEE Am. Ctrl. Conf. (1997), IL 2 (1997) 1096-1100.

[12] Rafael Martínez-Guerra, Ricardo Aguilar and Alexander Poznyak. A new robust sliding-mode observer design for monitoring in chemical reactors. Journal of Dynamic System, Measurement and Control, ASME Journal. 2004. 126, 473-478.

[13] Hu S and Wang J. Global asymptotic stability and global exponential stability of continuous time recurrent neural networks. IEEE Trans. Aut. Ctrl. 47, 5 (2002) 802-807.

[14] Schuler, H. And Schmidt, C. Calorimetric-state estimator for chemical reactors diagnosis and control: review of methods and applications. Chem. Eng. Sci. 47 (1992) 899-915.

[15] R. Aguilar-López, R. Martínez-Guerra, J. Mendoza-Camargo and M.I. Neria-González. Monitoring of an industrial wastewater plant employing finite-time convergence observer. J. of Chemical Technology \& Biotechnology. 81, 6, 2006. 
[16] R. Aguilar-López and R. Martínez-Guerra. Discrete algebraic estimator design for nonlinear Liouvillian systems with sampled output: Application to continuous bioreactor. Chemical Engineering Journal. 2006. 118, 23-28.

[17] R. Aguilar-López and R. Martínez-Guerra. State estimation for nonlinear systems under model unobservable uncertainties: Application to continuous reactor. 2005. Chemical Engineering Journal. 108, 139-144.

[18] Ricardo Aguilar-López and R. Maya-Yescas. State estimation for nonlinear systems under model uncertainties: A class of sliding-mode observers. Journal of Process Control. 2005, 15, 363-370.

[19] Neria-González I, Wang ET, Ramírez V, Romero JM, Hernández-Rodríguez C. Characterization of Bacterial Community Associated to Biofilms of Corroded Oil Pipelines from the Southeast of Mexico. Anaerobe 12 (2006) 122-133.

[20] Ravot G, Ollivier B, Magot M, Patel BKC, Crolet J-L, Fardeau M-L, and García J-L. Thiosulfate reduction, an important physiology feature shared by members of the order Thermotogales. Appl. Environ. Microbiol. 61 (1995) 2053-2055.

[21] Cord-Ruwisch R. A quick method for determination of dissolved and precipitated sulfides in cultures of sulfate-reducing bacteria. J. Microbiol. Meth. 4 (1985) 33-6.

[22] Kolmert $\AA$, Wikström $P$ and Hallberg K. A fast and simple turbidimetric method for the determination of sulfate in sulfate-reducing bacterial cultures. J. Microbiol. Meth. 41 (2000) 179184.

[23] Bailey JE and Ollis DF. Biochemical Engineering Fundamentals, $2^{\text {nd }}$ ed. McGraw Hill BC, Singapore (1986).

[24] Arcak M and Kokotović P. Observer-based control of systems with slop-restricted nonlinearities. IEEE Trans. Automat. Cont. 46 (2001) 1146-1150.

[25] Ibrir S. On-line exact differentiation and notion of asymptotic algebraic observers, IEEE Trans. Automat. Cont. 48 (2003) 2055-2060.

[26] Gauthier JP, Hammouri H and Othman S. A simple observer for nonlinear systems: application to bioreactors. IEEE Trans. Automat. Cont. 37 (1992) 875-880.

[27] G. Kreisselmeier and Engel R. Nonlinear observers for autonomous lipschitz continuous systems, IEEE Trans. Automat. Cont. 48 (2003) 451-464. 
Authors' Biography

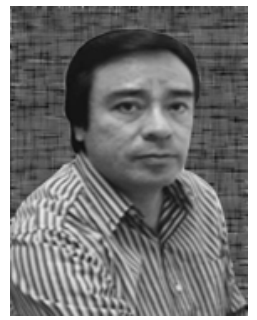

Ricardo Aguilar López

Born in Mexico City in 1964. He earns his BSc, MSc and PhD degrees from Universidad Autónoma Metropolitana in 1989, 1993 and 1998, respectively, all of them in chemical engineering. He was a postdoctoral fellow at Instituto Mexicano del Petróleo (2000); besides, he earns a Ph D in automatic control from CINVESTAV-IPN (2003). He works in robust observer design for nonlinear systems and process control. He is author and co-author of 83 papers published in international journals. Currently, he is a researcher at the Departamento de Biotecnología y Bioingeniería del Centro de Investigación y Estudios Avanzados del IPN and member of the Sistema Nacional de Investigadores since 1998 (level II, currently).

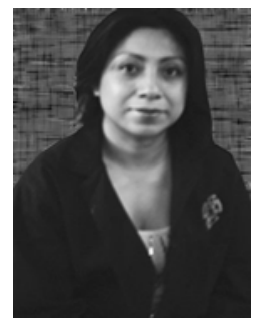

\section{María Isabel Neria González}

Born in Mexico City in 1971, she earns a degree in biochemical engineer from Universidad Autónoma Metropolitana (1999) and her M sc and Ph D degrees in microbiology science from Escuela Nacional de Ciencias Biológicas del IPN (2002 and 2006, respectively). She was a postdoctoral fellow at Departamento de Biotecnología y Bioingeniería del CINVESTAV-IPN and Microbiology Department of Provence University, France. She is author and/or coauthor of 11 published papers in indexed journals. Currently, she is a full-time professor at Tecnológico de Estudios Superiores de Ecatepec and candidate for national researcher at S.N.I.

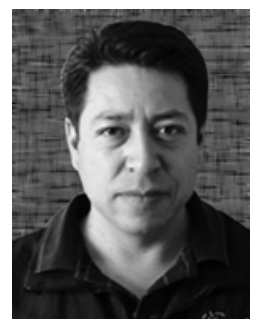

Ricardo Acevedo Gómez

Born in Mexico City, December 20 1969. He received his BSc degree from the Escuela Superior de Ingeniería Textil in Mexico City; his first degree studies were conducted at the Escuela Superior de Ingeniería Textil in linking with the Universidad Politécnica de Cataluña in Barcelona, Spain. Currently, 
he is pursuing doctoral studies in the Departamento de Biotecnología y Bioingeniería at the Centro de Investigaciones y Estudios Avanzados of the IPN (CINVESTAV-IPN), in Mexico City. His research experience involves the creation of a web page for troubleshooting related to electricity and magnetism, minimization of production costs in the making industry and detergency evaluation on different tinted and white cotton and polyester products. At present, $\mathrm{He}$ is carrying out research on variable estimation in biosystems using robust observers.

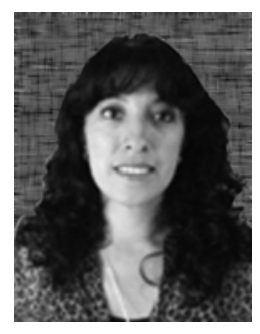

\section{Alma Rosa Domínguez Bocanegra}

Born in Mexico City in 1965. She earns a degree in biochemical engineering from Escuela Nacional de Ciencias Biológicas (1991) and the Ph D degree in biological sciences from Universidad Autónoma Metropolitana in 2003. She has been a research assistant in the Departamento de Bioingeniería y Biotecnología at the CINVESTAV-IPN, Mexico, since 1991 and a full-time professor at the Tecnológico de Estudios Superiores de Ecatepec since 1992. She visited the university of Almeria and the Instituto de Bioquímica y Biosíntesis Vegetal at the CSIC in Sevilla, Spain, in 2002 and 2004, respectively. She works in microalgae biotechnology with pigment production and water bioremediation. She is author and/or coauthor of 20 papers published in indexed journals. She has been a member of the Sistema Nacional de Investigadores since 2000, (level I, currently). 